\title{
Analyse cytogénétique du complexe Anopheles gambiae dans un village du Sud-Est du Burkina-Faso*
}

\author{
V Robert ${ }^{1 * *}$, V Petrarca ${ }^{2}$, P Carnevale $^{1}$, A Zoulani $^{1}, \mathrm{M} \mathrm{Coluzzi}^{2}$ \\ ${ }^{1}$ Antenne ORSTOM du Centre Muraz, BP 171, Bobo-Dioulasso, Burkina Faso; \\ ${ }^{2}$ Università di Roma, «La Sapienza», Istituto di Parassitologia \\ Piazzale Aldo Moro 5, 00185 Roma, Italia
}

(Reçu le 4 janvier 1989; accepté le 20 décembre 1989)

Résumé - L'analyse cytogénétique d'un échantillon du complexe Anopheles gambiae, prélevé dans le Sud-Est du Burkina-Faso, prouve que cette région est une zone de sympatrie entre $A$ arabiensis et $A$ gambiae. Aucun hybride entre ces 2 espèces jumelles n'a été observé sur 229 examens. Sur le plan génétique $A$ arabiensis se présente comme une espèce homogène où les croisements sont aléatoires. Par contre, pour l'espèce $A$ gambiae le déficit de certains génotypes conduit à définir 2 formes chromosomiques, $A$ gambiae Mopti et $A$ gambiae Savane, qui constituent deux populations caractérisées par un isolement reproductif partiel. La fréquence d'hybrides entre ces 2 formes chromosomiques dans l'échantillon est estimée à moins de $6 \%$. Au sein de chacune de ces formes chromosomiques, les croisements sont aléatoires. L'hypothèse de mécanismes précopulatoires d'isolement entre ces 2 populations est proposée.

complexe Anopheles gambiae / cytogénétique / équilibre d'Hardy-Weinberg / Afrique

Summary - Cytogenetic analysis of the Anopheles gambiae complex in a southeastern village of the Burkina-Faso. The cytogenetic analysis of a sample of the Anopheles gambiae complex from a village of south-eastern Burkina-Faso shows that A arabiensis and A gambiae are sympatric in this zone. No hybrid between these 2 sibling species was observed among 229 mosquitoes. From a genetic point of view, A arabiensis appears to be a panmictic population. On the contrary, in A gambiae, the deficiency of certain genotypes enables 2 chromosomal forms to be defined, A gambiae Mopti and A gambiae Savanna, which are characterized by partial reproductive isolation. The frequency of hybrids between the 2 chromosomal forms is evaluated at less than $6 \%$. Cross-breedings are random within each of these chromosomal forms. The hypothesis concerning precopulatory isolating mechanisms between those 2 populations is suggested.

Anopheles gambiae complex / cytogenetic / Hardy-Weinberg equilibrium / Africa

* Cette recherche a bénéficié d'une aide financière du Programme spécial de recherche et de formation pour les maladies tropicales PNUD/Banque Mondiale/OMS et de la Commission des Communautés Européennes, DGXII.

** Correspondance et tirés à part: antenne ORSTOM de l'OCEAC, BP 288, Yaoundé, Cameroun 


\section{INTRODUCTION}

Le complexe Anopheles gambiae Giles 1902 est probablement le plus étudié et le plus finement analysé des complexes d'espèces d'intérêt médical. On savait depuis les années 1940 qu'Anopheles gambiae sensu lato présentait une hétérogénéité dans ses capacités comme vecteur de paludisme dans différentes parties de l'Afrique (De Meillon, 1956). La découverte de populations à stades préimaginaux halophiles sur le pourtour de l'Afrique introduisait d'autres différences (Ribbands, 1944). L'emploi généralisé d'insecticides lors des campagnes de lutte antipaludique a suscité des comportements-réponses très variables selon les localités (Davidson, 1956) faisant suspecter l'existence de taxons différents. C'est en 1962 qu'éclata définitivement le taxon $A$ gambiae $s l$ grâce à la méthode des croisements avec des souches de référence (Davidson et Jackson, 1962; Davidson, 1962). À la suite de ces découvertes, des études de morphologie ont été reprises avec du matériel biologique identifié génétiquement; elles ont montré une similitude complète entre les différentes espèces sauf pour les espèces halophiles vis-à-vis des espèces dulcaquicoles (Coluzzi, 1964) ou pour certaines populations marginales (Chauvet et al, 1969; Zahar et al, 1970). La technique morphologique classique était inopérante et la technique mixiologique nécessitait trop de temps; une nouvelle technique était nécessaire pour déterminer les espèces et analyser plus avant leurs inter-relations.

L'étude cytogénétique des chromosomes polyténiques des glandes salivaires du quatrième stade larvaire (Frizzi et Holstein, 1956) et des cellules trophocytaires de l'ovocyte du stade de Cristophers III fin à IV début (Coluzzi, 1968) s'est avérée la plus performante et s'est généralisée. Ces études chromosomiques ont permis de définir cytotaxonomiquement les différentes espèces du complexe $A$ gambiae (Coluzzi, 1966; Coluzzi et Sabatini, 1967, 1968, 1969; Davidson et Hunt, 1973).

Ces espèces sont au nombre de 6. A gambiae sensu stricto (ancienne espèce A) et $A$ arabiensis (ancienne espèce $B$ ) sont les plus anthropophiles et les meilleurs vecteurs de paludisme, de filariose de Bancroft et d'arbovirose. En général, A gambiae domine en zone de forêt et de savane humide (Coz, 1973a). A arabiensis est plus zoophile et plus exophile qu' $A$ gambiae. Ces 2 espèces sont sympatriques dans la quasi totalité de la zone afrotropicale non méridionale. A quadriannulatus (ancienne espèce C) est strictement zoophile et est rencontré en Éthiopie et dans l'Est de l'Afrique méridionale. Les larves de ces 3 espèces vivent dans l'eau douce, contrairement à celles des trois suivantes. Les larves d'A bwambae (ancienne espèce $D$ ) vivent dans les sources d'eau minérale de la forêt de Semliki en Ouganda (White, 1985). Les larves d'A melas et d'A merus vivent en eau saumâtre respectivement sur le littoral Ouest et Est de l'Afrique. Ces 3 dernières espèces sont allopatriques entre elles mais sympatriques à la fois avec $A$ gambiae et/ou $A$ arabiensis (White, 1974).

Chacune de ces 6 espèces est manifestement protégée par d'efficaces mécanismes d'isolement reproductif. Ces mécanismes ne sont pas encore formellement identifiés mais certainement relatifs à des composants éthologiques, agissant avant la copulation (Coluzzi et al, 1985). Bien que la possibilité d'hybridation existe dans de nombreuses zones de sympatrie, les hybrides naturels entre ces 6 espèces sont très rares. Les hybrides obtenus par croisements expérimentaux sont le plus souvent viables. La stérilité est de règle seulement pour les mâles hybrides. 
Les études effectuées au Burkina-Faso (ex-Haute-Volta) par Coz (1973 a et b) ont démontré qu' $A$ gambiae et $A$ arabiensis sont sympatriques dans tout le pays et que leur fréquence relative est fonction des conditions climatiques. A gambiae domine dans la partie méridionale humide du pays, alors qu'A arabiensis est mieux représenté dans les steppes sahéliennes.

Des études plus récentes portant sur le polymorphisme génétique des membres de ce complexe en association avec un environnement anthropique, ont eu pour cadre le Nigeria (Coluzzi et al, 1979), la Sénégambie (Bryan et al, 1982; Petrarca et al, 1987) et le Mali (Touré et al, 1983). Il a été montré que l'étude des inversions chromosomiques donnait d'importantes informations sur le polymorphisme génétique des populations naturelles du complexe. Une partie de l'hétérogénéité génétique d'A gambiae $s s$ était liée à un processus de spéciation en cours dont les inversions chromosomiques en constituait un excellent marqueur. C'est ainsi qu'ont déjà été définies 5 formes chromosomiques, caractérisées par un isolement reproductif au moins partiel, nommées avec une nomenclature non linéenne: «Mopti», «Savane», «Bamako», «Bissau» et «Forêt» (Coluzzi et al, 1985).

Des recherches cytogénétiques sur $A$ gambiae et $A$ arabiensis ont déjà été conduites au Burkina Faso dans le Sud-Ouest (Robert et al, 1989) et dans le centre (Petraca et al, 1986). Aucune information n'est disponible sur le Sud-Est, c'est pourquoi un échantillon d'A gambiae $s l$ a été prélevé dans cette région et a été analysé.

\section{MATÉRIEL ET MÉTHODES}

\section{La zone d'étude}

La récolte de moustiques a été faite à Gagaré $\left(11^{\circ} 49^{\prime} \mathrm{N}, 00^{\circ} 13^{\prime} \mathrm{E}\right)$, petit village de la province du Gourma, à $35 \mathrm{~km}$ au Sud de Fada N'Gourma. C'est une zone de plateaux relativement secs recouverts de savane arborée de type soudanien. Il pleut en moyenne $900 \mathrm{~mm}$ d'eau par an pendant l'unique saison pluvieuse qui dure de juin à septembre. Le village est implanté à proximité d'un cours d'eau temporaire bordé d'une belle galerie forestière. Lors de l'enquête, la saison sèche était déjà installée et il ne restait plus, dans le lit sableux des cours d'eau de la région, que quelques flaques résiduelles en voie d'assèchement mais encore productives en $A$ gambiae $s$ l.

Les habitants sont des Peuls sédentaires qui vivent de la culture du mil pratiquée autour de leurs habitations, et de l'élevage (bovins, ovins, caprins et volailles). Le bétail rentre tous les soirs pour dormir à proximité immédiate des habitations.

L'habitat est très dispersé, il est constitué par des huttes hémisphériques à armature de branchage et à revêtement de paille tressée. Ces huttes constituent d'excellents gîtes de repos diurnes pour les moustiques en particulier pour A gambiae $s$.

\section{Méthode de capture}

La récolte au pyrèthre de la faune culicidienne intradomiciliaire a été effectuée entre $15 \mathrm{~h}$ et $18 \mathrm{~h}$, du 18 au 20 septembre 1984. La même dizaine de maisons, réparties sur moins d'un kilomètre carré a pu être visitée avec succès pendant 3 jours consécutifs. 
Ainsi l'échantillon prélevé possède de bonnes caractéristiques d'unité de lieu et de temps.

Les femelles semi-gravides d'A gambiae $s l$ ont été immédiatement triées et plongées dans un fixateur cytologique: le Carnoy (3/4 alcool éthylique absolu, $1 / 4$ acide acétique glacial). Ces moustiques ont été conservés à température ambiante pendant quelques jours puis placés à $-20^{\circ} \mathrm{C}$.

\section{Méthode d'analyse génétique}

Les chromosomes des cellules trophocytaires de l'ovocyte ont été préférés à ceux des glandes salivaires du quatrième stade larvaire car ils sont présents chez la femelle adulte, sont plus facilement accessibles et procurent une fréquence supérieure de préparations réussies. La technique de préparation des chromosomes polyténiques a été celle de Hunt (1973) et leur lecture a été faite au microscope à contraste de phase (figure 1) avec la nomenclature de Coluzzi et al (1979). Chacun des 3 chromosomes est désigné par un numéro. Le chromosome 1 est l'hétérochromosome; il est télocentrique. Les chromosomes 2 et 3 sont autosomaux et submétacentriques. Les bras de part et d'autre du centromère sont désignés par les lettres $R$ ou $L$ pour «right» ou «left». Les inversions sont désignées par des lettres minuscules sur chaque bras. L'arrangement standard de référence est notée + . Ce même symbole, avec une notation particulière, peut désigner l'arrangement standard d'une section du bras chromosomique; par exemple $2 R^{+a}$ désigne l'alternative standard de l'inversion $a$ du bras droit du chromosome 2. L'ensemble de tous les arrangements alternatifs d'une portion de bras chromosomique constitue un système d'inversion. Ces arrangements alternatifs sont d'autre part à l'origine d'une série de caryotypes exclusifs les uns des autres.

Les arrangements chromosomiques observés dans la préparation sont des inversions paracentriques qui sont soit fixées dans le taxon et utilisées dans la diagnose spécifique, soit polymorphiques et utilisées dans l'analyse génétique. L'accord entre les fréquences des caryotypes observés et attendus selon la loi d'Hardy-Weinberg a été testé par un test de $\chi^{2}$. Pour des systèmes d'inversion avec 2 arrangements alternatifs (1 degré de liberté) on a appliqué la correction de Yates lorsqu'un des caryotypes montrait un effectif attendu inférieur à 5 . Dans le cas d'un nombre de degré de liberté supérieur à 1 , les classes dont les effectifs attendus étaient inférieurs à 1 ont été cumulées et le nombre des degrés de liberté a été modifié en conséquence (Ayala et Kiger, 1984). Les groupes d'individus pour lesquels l'équilibre d'Hardy-Weinberg s'est vérifié ont été considérés comme appartenant à un ensemble génétique homogène dont le statut minimum est celui d'une population. En revanche, si cette hypothèse est rejetée, l'existence de plus d'une unité panmictique dans l'échantillon sera recherchée.

Le plus souvent le génotype d'un individu est directement déduit de sa préparation chromosomique. Toutefois, une partie des polyhétérozygotes ont la même configuration cytologique (par exemple $b c / b d$ et $b c d / b$ ) et leur discrimination n'a pas toujours été possible avec l'étude de la séquence des bandes sur les 2 chromosomes homologues appariés. L'attribution de génotype dans l'une ou l'autre des classes alternatives a été fondée sur la fréquence relative des caryotypes et des arrangements indiscutables car à l'état homozygote. 


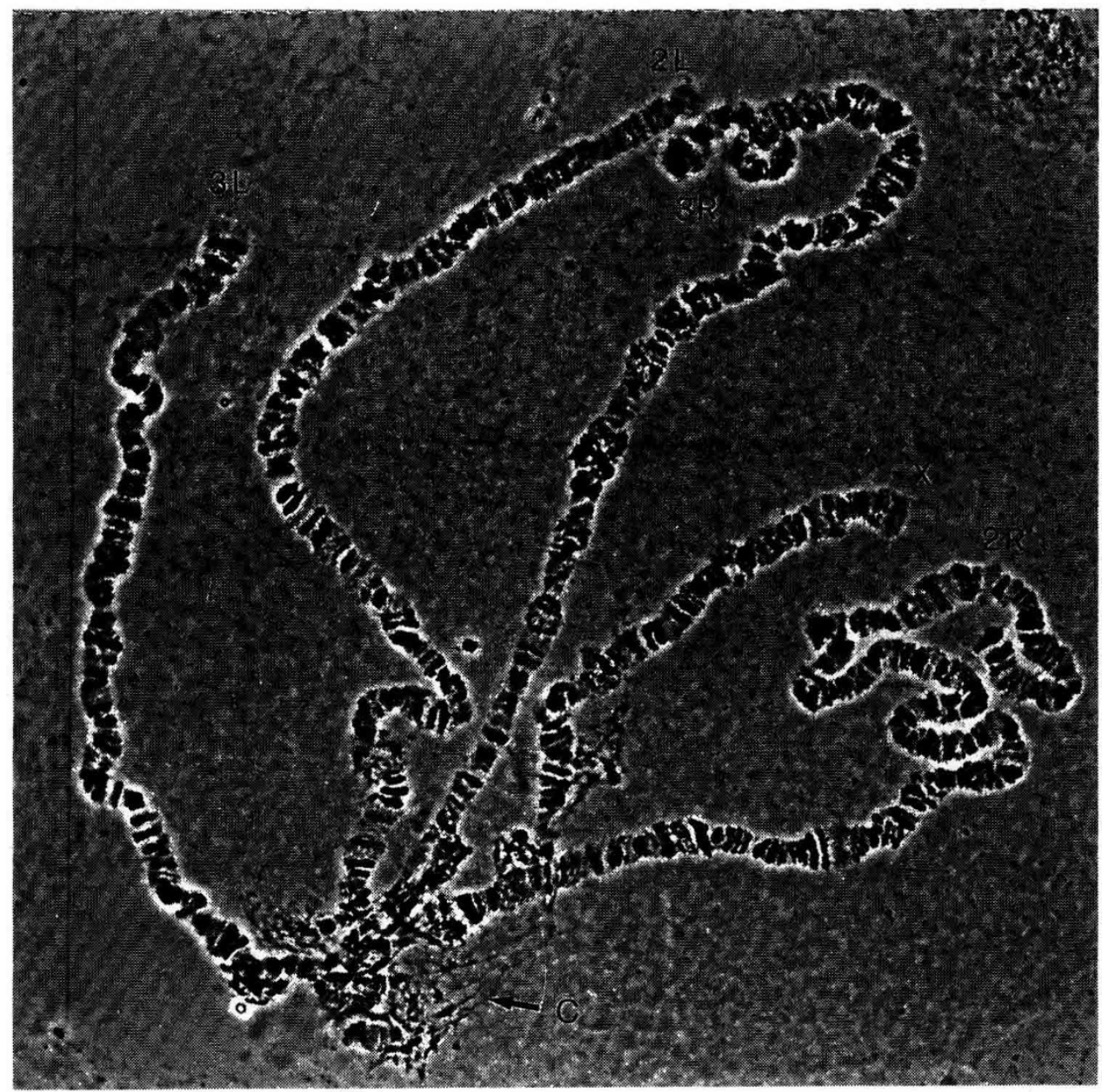

Fig 1. Complément polyténique d'une cellule trophocytaire de l'ovocyte d'un Anopheles gambiae ss de la forme chromosomique Mopti présentant un caryotype homozygote $2 R$ $b c / b c$ et $2 L a / a . \mathrm{C}=$ centromère.

\section{RÉSULTATS}

Sur les 247 femelles semi-gravides récoltées, 229 (soit 92,7\%) ont fourni des préparations chromosomiques interprétables. L'examen cytomorphologique du chromosome $X$ a permis de reconnaître 138 A arabiensis (soit 60\%) et 91 A gambiae (soit 40\%). Aucun hybride entre ces 2 espèces n'a été observé. 
Le polymorphisme chromosomique d'Anopheles arabiensis

\section{Observations}

Les inversions observées portent sur le bras $2 R$ et $3 R$. Pour le bras $2 R$, on observe des inversions dont la position autorise leurs classements en 3 systèmes (figure 2, tableau I):

2Ra: avec les arrangements ${ }^{+a}, a$ et $b f$;

$2 R b$ : avec les arrangements ${ }^{+b}, b, b c$, be et $b f$;

$2 R d^{1}$ : avec les arrangements ${ }^{+d 1}, d^{1}$, be et $b f$.

Pour le bras $3 R$ on observe les arrangements alternatifs ${ }^{+a}$ et $a$.

\section{Analyse génétique}

En utilisant ces données, on calcule selon le roi d'Hardy-Weinberg les effectifs attendus des associations d'arrangements chromosomiques pour chaque système d'inversion. Leur comparaison avec les effectifs observés montre que pour les systèmes $2 R a, 2 R b 2 R d^{1}$ et $3 R a$, les différences ne sont pas significatives et sont compatibles avec l'hypothèse de croisements aléatoires chez $A$ arabiensis (tableau I).
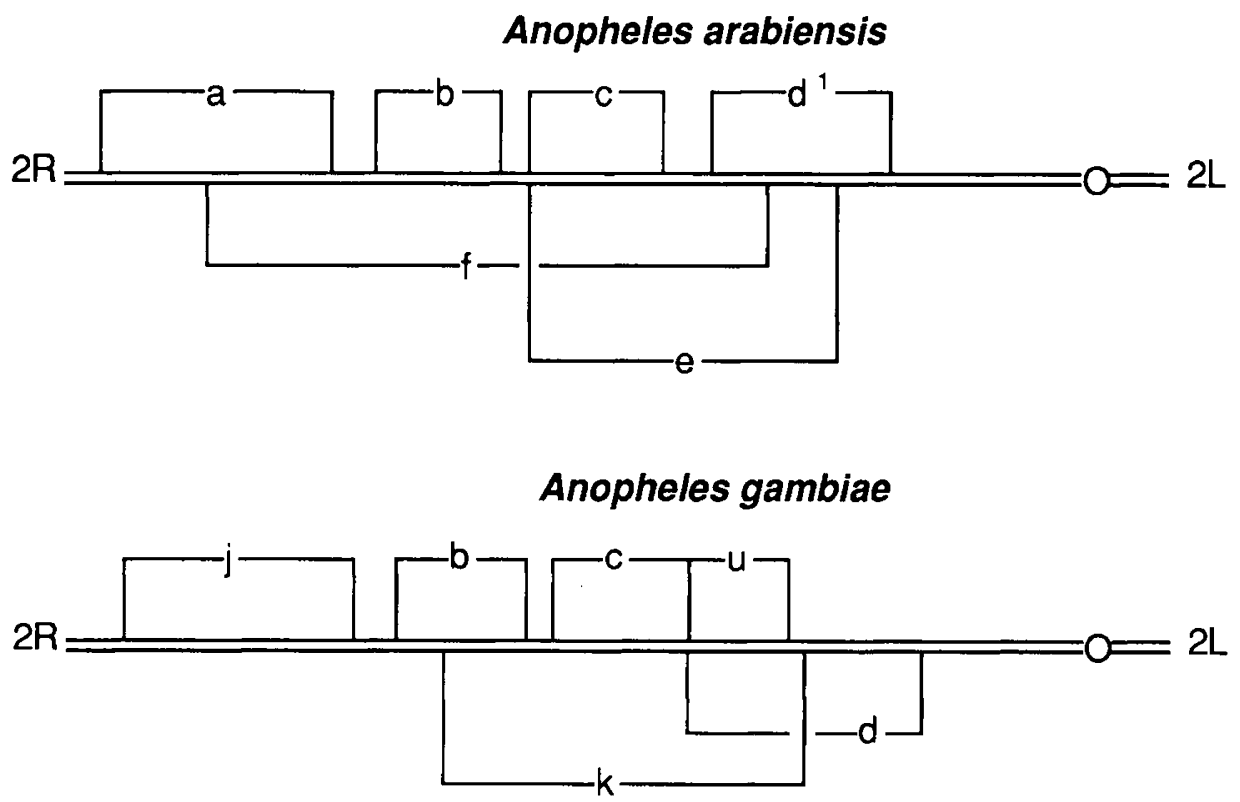

Fig 2. Schémas des inversions rencontrées sur le bras chromosomiques $2 R$ d'Anopheles arabiensis et d'A gambiae dans le village de Gagaré (Burkina-Faso). 
Table I. Caryotypes observés et attendus pour 4 systèmes d'inversions chromosomiques d'Anopheles arabiensis.

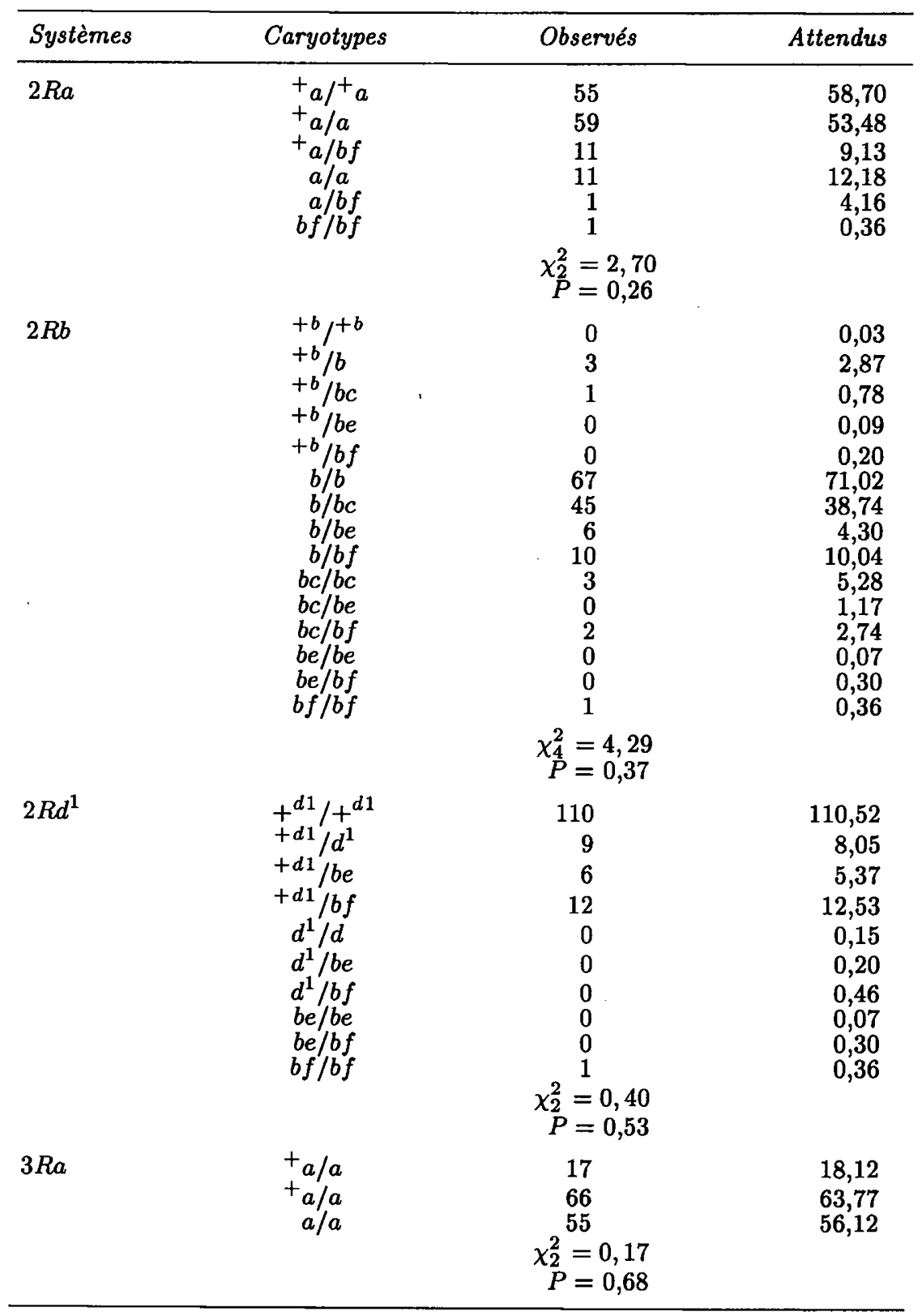




\section{Le polymorphisme chromosomique d'A gambiae}

\section{Observations}

Les inversions observées portent sur les bras $2 R$ et $2 L$. Pour le bras $2 R$ on observe des inversions dont la position autorise leur classement en 2 systèmes (figure 2, tableau II):

$2 R b$ : avec les arrangements ${ }^{+b}, b$ et $b c$;

$2 R d$ : avec les arrangements ${ }^{+d}, d$ et $u$.

Un individu portant un caryotype $2 R j b k / b$ ou $2 R j b / b k$ a été aussi observé, mais en raison de sa singularité il n'a pas été comptabilisé.

Pour le bras $2 L$ on observe les arrangements alternatifs ${ }^{+a}$ et $a$.

\section{Analyse génétique}

Analyse globale de la variabilité chromosomique d'A gambiae

A partir de ces données, les effectifs attendus des associations d'arrangements chromosomiques sont calculés pour les systèmes $2 R b$ et $2 R d$. La différence entre ces effectifs et les effectifs observés est très significative pour le système $2 R b$; elle ne l'est pas pour le système $2 R d$ (tableau II). L'hypothèse selon laquelle les inversions chromosomiques s'associent selon les lois du hasard est donc rejetée chez A gambiae.

Table II. Caryotypes observés et attendus pour 2 systèmes d'inversions chromosomiques d'Anopheles gambiae

\begin{tabular}{cccr}
\hline Systèmes & Caryotypes & Observés & Attendus \\
\hline $2 R b$ & $+b /+b$ & 5 & 4,67 \\
& $+b / b$ & 6 & 18,22 \\
& $+b / b c$ & 25 & 13,44 \\
& $b / b$ & 28 & 17,78 \\
& $b / b c$ & 18 & 26,22 \\
& $b c / b c$ & 8 & 9,67 \\
& & $\chi_{3}^{2}=26,9$ & \\
& & $<<<01$ & \\
& $+d /+d$ & 65 & 65,88 \\
& $+d / d$ & 16 & 15,40 \\
& $+d / u$ & 8 & 6,84 \\
& $d / d$ & 1 & 0,90 \\
& $u / u$ & 0 & 0,18 \\
& $u / d$ & 0 & 0,80 \\
& & $\chi_{1}^{2}=0,64$ & \\
\hline
\end{tabular}




\section{Prise en compte de l'existence de 2 unités infraspécifiques}

La majorité des génotypes a été déterminée sans ambiguïté mais un certain nombre de cas prêtent à discussion sur le bras chromosomiques $2 R$ :

- le triple hétérozygote $b c u$ peut correspondre théoriquement à 4 caryotypes: $b c u /+, b c / u, b u / c, b / c u$. Les 5 individus de ce type ont tous été attribués à $b c / u$. En effet les arrangements $b c u$ et $c u$ ont été observés exceptionnellement au BurkinaFaso, l'arrangement $b u$ est rare et $b c$ fréquent dans notre échantillon, enfin pour quelques préparations particulièrement réussies on a pu vérifier que les inversions $b$ et $c$ sont portées par un même chromosome et que $u$ est sur l'autre;

- les génotypes $b c d / b$ et/ou $b c / b d$ regroupent 13 individus. En fonction des proportions des arrangements $b, b c, b d$ et $b c d$ et de la certitude de l'existence dans cette population des génotypes $b c d / b c d$ et $b d / b$, on a décidé d'en affecter 11 au génotype $b c d / b$ et 2 au génotype $b c / b d$;

- les 2 individus triple-hétérozygotes pour les inversions $b, c$ et $d$ ont été classés dans le génotype $b c d /^{+}$. Les autres génotypes possibles $b c / d, b / c d$ et $b d / c$, sont beaucoup moins probables, surtout les 2 derniers;

- le génotype $b c / b u$ a été préféré à $b c u / b$, moins probable, à cause de la plus grande fréquence de $b u$ par rapport à celle de $b c u$ dans le reste du Burkina-Faso.

En conformité avec les travaux de Touré et al (1983), Coluzzi et al (1985), Petrarca et al (1986) et Robert et al (1989) on a défini des groupes d'arrangements chromosomiques pour tenter de retrouver des ensembles génétiquement homogènes à un niveau infraspécifique. Les $90 \mathrm{~A}$ gambiae de notre échantillon ont été répartis en fonction de leurs arrangements parmi 2 formes chromosomiques: la forme «Savane» caractérisée par l'inversion $b$ et la forme «Mopti» caractérisée par les inversions $b c$ et $u$ (tableau III).

Le génotype standard $+/^{+}$peut être considéré comme appartenant soit à la forme Savane soit à la forme Mopti. Les 3 individus de ce génotype ont été répartis en proportion des effectifs des formes, soit 2 Savane et 1 Mopti.

Finalement l'échantillon comprend 50 individus (soit 55,6\%) attribués à la forme Savane, 33 (soit 36,7\%) à la forme Mopti et seulement 7 (soit 7,8\%) restent non attribués. Parmi ces 7 non classés les $4 b c / b$ et les $2 b c / b d$ sont à interpréter comme des hybrides issus de croisements entre les 2 formes chromosomiques.

L'hypothèse de la panmixie au sein de chaque forme chromosomique a été testée sur les systèmes $2 R b$ et $2 R d$. Pour les 2 formes et pour les 2 systèmes on a calculé la fréquence des arrangements chromosomiques puis les effectifs attendus des différents arrangements. La comparaison des effectifs observés et des effectifs attendus (tableau IV) n'a pas mis en évidence de différence motivant un rejet de l'hypothèse de croisements aléatoires à l'intérieur de chaque forme prise isolément. Chaque forme chromosomique correspond donc à une véritable unité génétique superposable à une entité taxonomique.

\section{DISCUSSION}

Le village de Gagaré dans le Sud-Est burkinabé est une zone de sympatrie pour $A$ arabiensis et $A$ gambiae. 
Tableau. Nombre et frequence $(x)$ des caryotypes d'inversion du bras $2 R$ in toto des 90 Anopheles qambiae recoltes a Gagaré et leur regroupement en formes chromosomiques Savane et Mopti ( 1 caryotype présentant les arrangements $2 R$ j et $2 R$ bk a eté négligé).

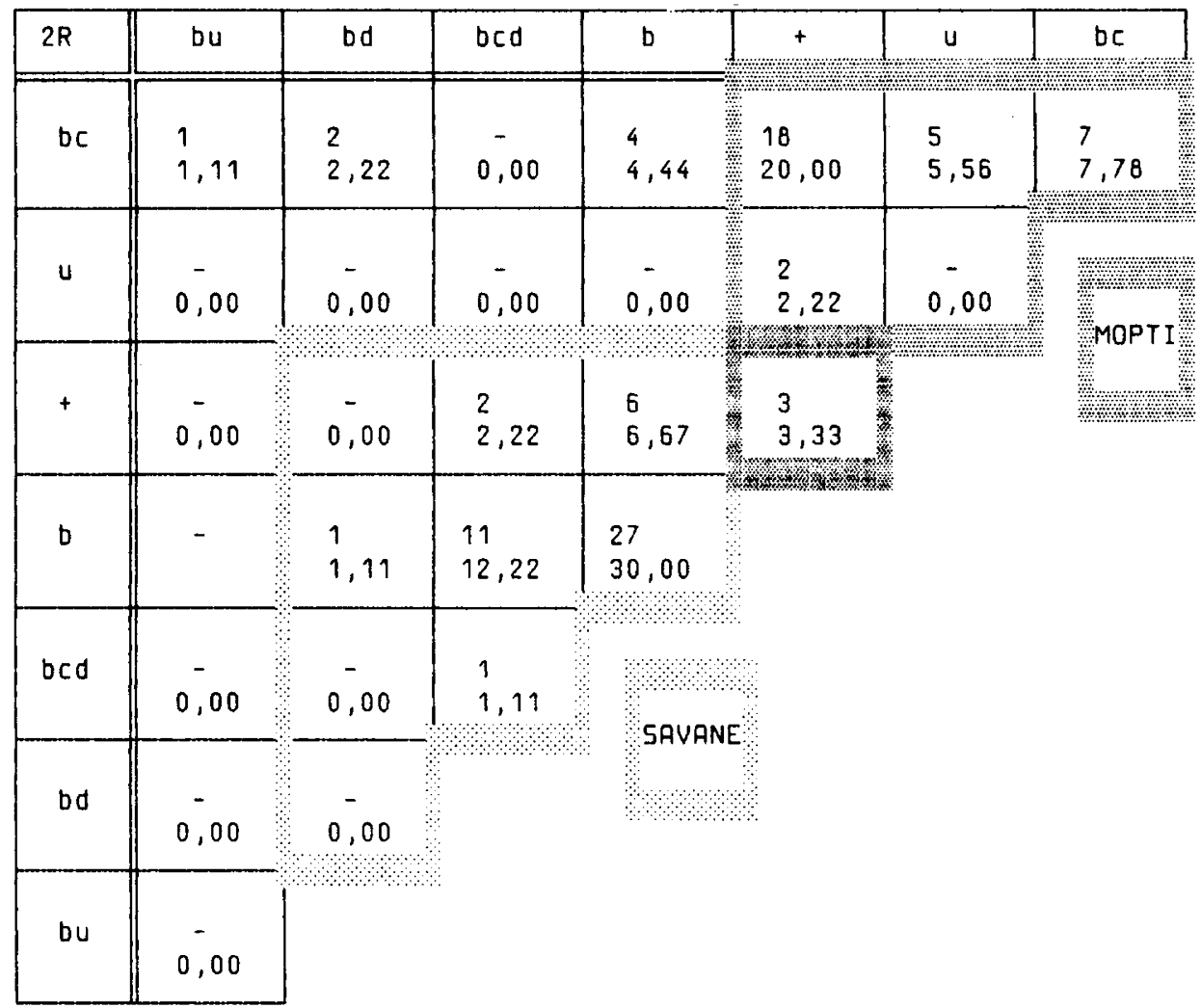

La fréquence relativement élevée d'A arabiensis dans notre échantillon est probablement consécutive à la présence nocturne de bovins dans le village et à la date du prélèvement en début de saison sèche, à un moment où les densités relatives de cette espèce sont souvent élevées (Rishikesh et al, 1985).

Parmi les $A$ gambiae 2 formes chromosomiques sont observées: Savane et Mopti. $A$ gambiae Savane, par l'existence d'un polymorphisme $2 R b / b c d$, est à rapprocher des A gambiae Savane du Nigeria (Coluzzi et al, 1979; Di Deco et al, 1980). Au Burkina-Faso central et occidental (Petrarca et al, 1986); Robert et al, 1989) et au Sénégal (Petrarca et al, 1987), cette forme chromosomique est surtout caractérisée par l'arrangement $2 R b$ avec une faible fréquence de ${ }^{+b}$. Selon Coluzzi et al (1985, article où une discussion approfondie porte sur les formes chromosomiques d' $A$ gambiae), au Mali et en Guinée les populations d'A gambiae Savane sont différenciées de celles du Burkina-Faso par la présence supplémentaire des arrangements $2 R b c u$ et $2 R c u$. La forme Mopti d'A gambiae, typique du delta intérieur du fleuve Niger, est caractérisée par le polymorphisme $2 R b c / u$. Cette forme semble avoir une aire de répartition s'étendant jusqu'aux zones non inondables du Sud-Est du Burkina- 
Table IV. Effectifs observés et attendus des 50 Anopheles gambiae Savane et 33 A gambiae Mopti prélevés dans le village de Gagaré (Burkina-Faso).

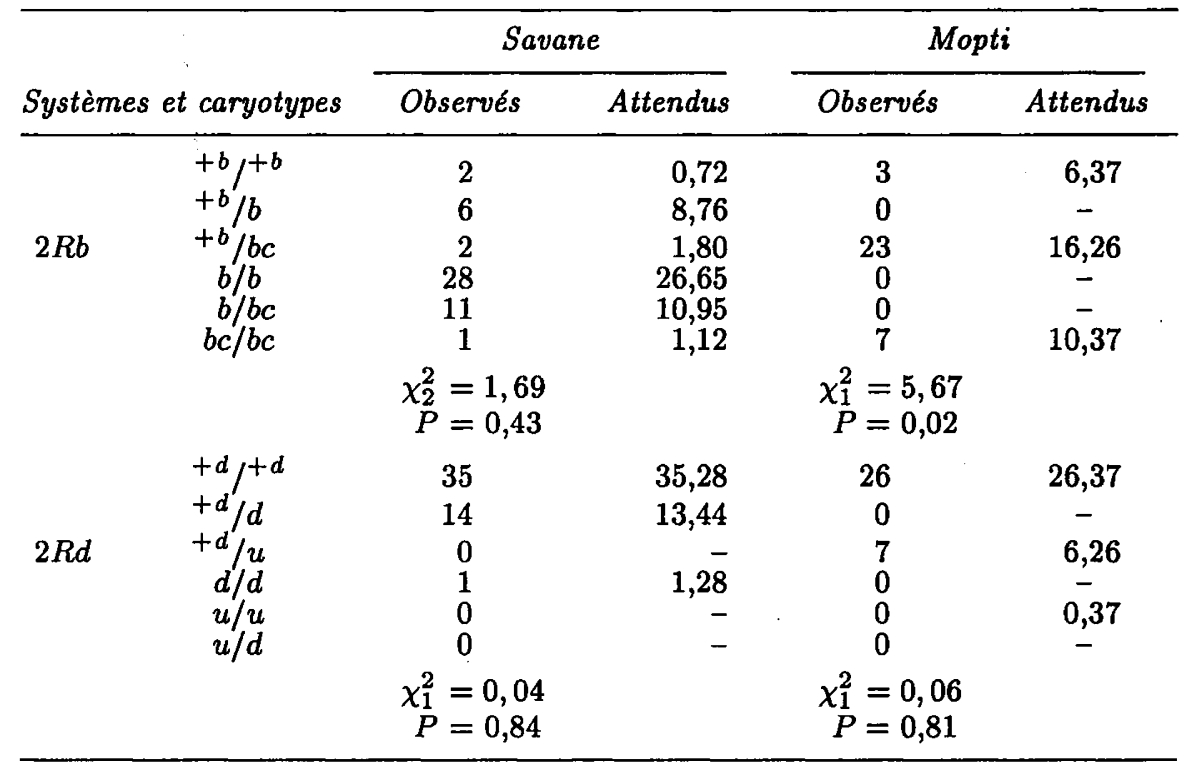

Faso. La fréquence relative de l'inversion $2 R u$ par rapport à l'inversion $2 R b c$ est toutefois beaucoup plus élevée à Gagaré (78\%) que dans le Sud-Ouest du BurkinaFaso (12\%) et le Sud du Mali, mais ceci peut être dû au fait que notre échantillon a été prélevé en début de saison sèche c'est-à-dire à une période de l'année où la fréquence de $2 R b c$ est élevée et où celle de $2 R u$ est basse; l'observation des variations saisonnières et adaptatives des fréquences de ces arrangements est voisine de celle de Dobzhansky (1943) sur Drosophila pseudoobscura. L'arrangement 2La est fixé chez Mopti et presque fixé chez Savane où seulement 2 caryotypes $2 L+a / a$ associés chacun à $2 R b / b$ ont été observés.

La position au rang d'espèce d' $A$ arabiensis et d' $A$ gambiae est désormais bien établie (Coluzzi et al, 1979). En revanche, la position taxonomique d'A gambiae Savane et d'A gambiae Mopti reste à déterminer; elle est nécessairement infraspécifique à cause d'une fréquence d'hybride non négligeable.

Pour expliquer ces degrés différents d'isolement génétique on peut avancer l'hypothèse de l'existence de barrières précopulatrices fortes entre $A$ arabiensis et $A$ gambiae et moins fortes entre $A$ gambiae Savane et $A$ gambiae Mopti (Coluzzi et al, 1985). Nous sommes convaincus que c'est l'hypothèse à retenir bien que les mécanismes de ces isolements reproductifs précopulatoires soient encore à élucider.

Le comportement particulier des membres de ce complexe dans leurs relations avec l'homme, donc dans la transmission du paludisme, renforce la nécessité de poursuivre de telles études cytogénétiques. 


\section{REMERCIEMENTS}

Les auteurs remercient Monsieur J Feingold et les 2 lecteurs anonymes pour les améliorations qu'ils ont apportées au manuscrit.

\section{RÉFÉRENCES}

Ayala J, Kiger JA Jr (1984) Modern Genetics. The Benjamin/Cummings Publishing Company

Bryan JH, Di Deco MA, Petrarca V, Coluzzi M (1982) Inversion polymorphism and incipient speciation in Anopheles Gambiae ss in The Gambia, West Africa. Genetica 59, 167-176

Chauvet G, Davidson G, Dejardin J (1969) Validité d'une méthode chétotaxique de distinction des larves d'espèces $\mathrm{A}$ et $\mathrm{B}$ du complexe Anopheles gambiae à Madagascar. Cah ORSTOM Sér Entomol Méd Parasitol 7, 1, 51-60

Coluzzi M (1964) Morphological divergences in the Anopheles gambiae complex. Riv Malariol 43, 197-232

Coluzzi M (1966) Osservazioni comparative sul cromosoma X nelle specie A e B del complesso Anopheles gambiae. Rend Acad Naz Lincei 40, 671-678

Coluzzi M (1968) Cromosomi politenici delle cellule nutrici ovariche nel complesso gambiae del genere Anopheles. Parassitologia (Rome) 10, 179-184

Coluzzi M, Sabatini A (1967) Cytogenetic observations on species A and B of the Anopheles gambiae complex. Parassitologia (Rome) 9, 73-88

Coluzzi M, Sabatini A (1968) Cytogenetic observations on species C of the Anopheles gambiae complex. Parassitologia (Rome) 10, 155-165

Coluzzi M, Sabatini A (1969) Cytogenetic observations on the salt water species, Anopheles merus and Anopheles melas, of the gambiae complex. Parassitologia (Rome) 11, 177-187

Coluzzi M, Petrarca V, Di Deco MA (1985) Chromosomal inversion intergradation and incipient speciation in Anopheles gambiae. Bol Zool 52, 45-63

Coluzzi M, Sabatini A, Petrarca V, Di Deco MA (1979) Chromosomal differentiation and adaptation to human environments in the Anopheles gambiae complex. Trans $R$ Soc Trop Med Hyg 73, 483-497

$\mathrm{Coz} \mathrm{J}$ (1973a) Contribution à l'étude du complexe $A$ gambiae. Répartition géographique et saisonnière en Afrique de l'Ouest. Cah ORSTOM Sér Entomol Méd Parasitol 11, 1, 3-31

Coz J (1973b) Contribution à la biologie du complexe Anopheles gambiae Giles en Afrique occidentale. Cah ORSTOM Sér Entomol Méd Parasitol 11, 1, 33-40

Davidson G (1956) Insecticide resistance in Anopheles gambiae Giles: a case of simple Mendelian inheritance. Nature 178, 861-863

Davidson G (1962) Anopheles gambiae complex. Nature 196, 907 
Davidson $G$, Hunt R (1973) The crossing and chromosome characteristics of a new sixth species in the Anopheles gambiae complex. Parassitologia (Rome) 15, 121-128

Davidson G, Jackson CE (1962) Incipient speciation in Anopheles gambiae Giles. Bull Org Mond Santé 27, 303-305

De Meillon B (1956) Aspects of malaria vector research in Africa. Bull WHO 847-851

Di Deco MA, Petrarca V, Villani F, Coluzzi M (1980) Recombination and linkage disequilibria between chromosome-2 inversions in Anopheles gambiae ss. Proceedings of the 3rd Europ Multicoll Parasit, Cambridge 7-13 Sept 1980, Abs 87

Dobzhansky Th (1943) Temporal changes in the composition of populations of Drosophila pseudoobscura. Genetics 28, 162-186

Frizzi G, Holstein M (1956) Étude cytogénétique d'Anopheles gambiae. Bull W $\mathrm{H} O 15,425-435$

Hunt RH (1973) A cytological technique for the study of Anopheles gambiae complex. Parassitologia (Rome) 15, 137-139

Petrarca V, Petrangeli G, Rossi P, Sabatinelli G (1986) Étude chromosomique d'Anopheles gambiae et Anopheles arabiensis à Ouagadougou (Burkina-Faso) et dans quelques villages voisins. Parassitologia (Rome) 28, 41-61

Petrarca V, Vercruysse J, Coluzzi M (1987) Observations on the Anopheles gambiae complex in the Senegal River Basin, West Africa. Med Vet Entomol 1, 303-312

Ribbands CR (1944) Differences between Anopheles melas (A. gambiae var. melas) and Anopheles gambiae. The larval pecten. Ann Trop Med Parasitol 38, 85-87

Rishikesh N, Di Deco MA, Petrarca V, Coluzzi M (1985) Seasonal variations in indoor resting Anopheles gambiae and Anopheles arabiensis in Kaduna, Nigeria. Acta Trop 42, 165-170

Robert V, Petrarca V, Carnevale P, Ovazza L, Coluzzi M (1989) Étude cytogénétique du complexe Anopheles gambiae dans la région de Bobo Dioulasso (Burkina-Faso). Ann Parasitol Hum Comp 64, 290-311

Touré YT, Petrarca V, Coluzzi M (1983) Nuove entità del complesso Anopheles gambiae in Mali. Parassitologia (Rome) 25, 367-370

White GB (1974) Anopheles gambiae complex and disease transmission in Africa. Trans $R$ Soc Trop Med Hyg 68, 278-301

White GB (1985) Anopheles bwanbae sp n; a malaria vector in the Semliki Valley, Uganda, and its relationships with other sibling species of the $A$ gambiae complex (Diptera, Culicidae) Systematic Entomol 10, 501-522

Zahar AR, Hills M, Davidson G (1970) An attempt to group fresh water species of the Anopheles gambiae complex by some morphological larval and adult characters. Parassitologia (Rome) 12, 31-46 